\title{
Subcortical Hyperexcitability in Migraineurs: A High-Frequency Oscillation Study
}

\author{
Kuan-Lin Lai, Kwong-Kum Liao, Jong-Ling Fuh, Shuu-Jiun Wang
}

\begin{abstract}
Objective: An abnormal central nervous system excitability level was found in patients with migraine. Whether it is hyper- or hypo-excitable is still debated. This study aimed to compare the somatosensory high-frequency oscillations (HFOs), which reflected subcortical excitability (early phase) and intracortical inhibition (late phase), between patients with migraine and control subjects. Methods: HFOs were recorded from C3'-Fz, using a 500-1000 Hz frequency filter after stimulation at right median nerves at the wrists, and divided into early and late phases based on the N20 peak. Fifty-nine untreated patients (n=24 during ictal period; n=35, interictal) and 22 controls finished the study. Results: In early HFOs, patients both during ictal and interictal periods had higher maximal amplitudes $(p=0.039)$ and area-under-curve $(p=0.029)$ than those of the controls. Regarding the late HFOs, there were no significant differences among these groups. Conclusion: Our study suggests a hyper-excitable state in the subcortical regions in patients with migraine both during interictal and ictal periods.
\end{abstract}

RÉSUMÉ: Hyperexcitabilité sous-corticale chez les migraineux : une étude d'oscillations à haute fréquence. Objectif : Un niveau anormal d'excitabilité du système nerveux central a été observé chez les patients atteints de migraine. Que ce soit de l'hyper ou de l'hypo excitabilité demeure un sujet de controverse. Le but de cette étude était de comparer les oscillations somatosensitives à haute fréquence (OHF), reflétant l'excitabilité souscorticale (phase précoce) et l'inhibition intra corticale (phase tardive), entre des patients atteints de migraine et des sujets témoins. Méthode : Les OHF ont été enregistrées de C3'-FZ, avec un filtre de fréquence 500-1000 Hz après stimulation du nerf médian droit au poignet, et séparées en phase précoce et tardive selon le pic N20. Cinquante-neuf patients non traités $(n=24$ pendant une crise ; $n=35$ entre les crises) et 22 sujets témoins ont complété l'étude. Résultats : En phase précoce d'OHF, les patients, qu'ils soient ou non en période de crise, avaient des amplitudes maximales $(\mathrm{p}=0,039)$ et une aire sous la courbe $(\mathrm{p}=0,029)$ plus élevées que celles des sujets témoins. Quant à la phase tardive d'OHF, il n'y avait pas de différence entre les groupes. Conclusion : Notre étude suggère qu'il existe un état d'hyperexcitabilité dans les régions sous-corticales chez les patients atteints de migraine, tant en période de crise qu'entre les crises.

Can J Neurol Sci. 2011; 38: 309-316

The pathophysiology of migraine remains largely undetermined. Current evidence suggests that activation of the trigeminovascular system (TGVS) is responsible for the generation of pain, and cortical spreading depression is the most probable primary event that triggers the activation of $\mathrm{TGVS}^{1}$. Since cortical spreading depression is preceded by transient synchronized neuronal excitation ${ }^{2}$, an altered state of brain excitability may be one of the fundamental pathophysiological mechanisms of migraine ${ }^{3}$. Thus, during the past decade, many electro-physiological studies have addressed the issue on interictal and ictal brain excitability in patients with migraine including somatosensory, visual and motor cortices by somatosensory evoked potential $(\mathrm{SEP})^{4,5}$, visual evoked potential (VEP) $)^{6,7}$ and transcranial magnetic stimulation (TMS $)^{8,9}$. However the results are conflicting and whether the brain is in a hyper- or hypo-excitable state in patients with migraine has been inconclusive.

Migraine is considered a disorder of sensory processing disturbance with altered perception of normality ${ }^{10}$. The pain process is likely to be a combination of activation of the nociceptors of the TGVS, and the facilitation or lack of inhibition of these signals in their pathway to the cortex ${ }^{11}$. Along the pain-transmission pathway, both the brainstem and the thalamus can exert their modulatory effects. The crucial role of the brainstem in the modulation of pain transmission in migraine pathophysiology is well documented ${ }^{12,13}$; whereas the role of the thalamus in migraine pathogenesis is less studied.

Somatosensory evoked potential is a method used to evaluate the function and integrity of the somatosensory pathway. Recent studies on SEP have disclosed several brief wavelets

From the Department of Neurology (KLL), Taipei Municipal Gandau Hospital; Department of Neurology (KLL, KKL, JLF, SJW), Neurological Institute, Taipei Veterans General Hospital; Department of Neurology (KLL, KKL, JLF, SJW), National Yang-Ming University School of Medicine, Taipei, Taiwan. Received June 14, 2010. Final Revisions Submitted August 26, 2010 Correspondence to: Shuu-Jiun Wang, Department of Neurology, Neurological Institute, Taipei Veterans General Hospital, No. 201, Shih-Pai Road, Section 2, Taipei, Taiwan, 112. 
superimposed on the N20 and P40 primary cortical response, following stimulation of the median nerve or the tibial nerve ${ }^{14-16}$. Because these inflections are of much higher frequencies (about $500-1000 \mathrm{~Hz}$ ) and smaller amplitudes than the N20 (about 20$50 \mathrm{~Hz}$ ), they are designated as "high-frequency oscillations" (HFOs). More recently, the generators of HFOs have been found to be of two origins. Using dipole source analysis in multichannel electroencephalogram (EEG) study, Gobbelé et al $^{17-19}$ demonstrated that the early phase of the HFOs originated from the deep thalamocortical fibers close to the thalamus. The late phase of the HFOs probably originated from intracortical GABAergic (gamma aminobutyric acid) inhibitory interneurons ${ }^{20,21}$, although some other generators have also been suggested, e.g., terminal thalamocortical fibers ${ }^{17,18}$, or pyramidal chattering cells ${ }^{22}$.

Since HFOs explore both the excitatory thalamocortical pathway from the thalamic relay nucleus, and probably an inhibitory intracortical activity, they can be used to study the pathophysiology of migraine. Two recent studies have addressed the issue on HFOs in patients with migraine and normal subjects $^{23,24}$. However, the results of the two studies were not completely the same. Sakuma et $\mathrm{al}^{23}$ demonstrated that the amplitudes of HFOs were significantly diminished in migraineurs at the interictal period compared to controls. Coppola et $\mathrm{al}^{24}$ found that the amplitudes and area-under-curve (AUC) of early HFOs in patients with migraine were decreased at their interictal period, but normalized at the ictal period. Regarding the late HFOs, there was no significant difference between patients and controls. In this study, we aim to examine both the conventional broad-band SEPs and HFOs in patients with migraine at ictal and interictal periods.

\section{Materials AND Methods}

\section{Subjects}

This study recruited both patients with migraine from the Headache Clinic of Taipei Veterans General Hospital and sexand age-matched control subjects. The diagnoses of migraine without aura (MO) and migraine with aura (MA) were based on the criteria proposed by the International Classification of Headache Disorders, 2nd edition (ICHD-2) (code 1.1 and 1.2) $2^{25}$. This study recruited only patients with episodic migraine; therefore, patients with more than 15 headache days per month were excluded. In addition, subjects on migraine prophylactic agents or hormone therapy, which might alter the brain excitability, were excluded. Post recruitment, all study subjects were required to keep a headache diary. Every subject completed a semi-structured questionnaire on demographics, medical history, medication behavior, and headache and aura profile during their first visit. Acute treatment was not allowed within three days prior to the examinations. The control subjects were colleagues, their relatives, or friends who did not have past or family histories of migraine and denied any headache attack in the past year. All of the subjects included in this study were naive to the SEP examination.

Both patients and control subjects received a face-to-face interview and physical and neurological examinations. The hospital's Institutional Review Board approved the study protocol and each participant provided written informed consent. The time interval (number of days) between HFO recordings (denoted as Day 0) and the most recent migraine attack prior to or after the measurement was determined in all patients based on their headache diaries. Patients who did not have migraine attack within a period of three days before (Days -1 to -3 ) and after (Days 1 to 3 ) the HFO recordings (Day 0) were classified as interictal, while those with migraine attacks within 24-hours (Day -1 to 1 ) were classified as ictal. Selection of the time window for the interictal and ictal periods was based on earlier studies $^{9,23,24}$

\section{SEP and $\mathrm{HFO}$ recordings}

For SEP recording (Neuropack M1, Nihon Kohden, Tokyo, Japan), subjects were asked to lay supine on a bed, and were instructed to keep alert with their eyes closed. Since HFOs are influenced by sleep and arousal changes ${ }^{16,26,27}$, we simultaneously recorded EEG by means of two recording electrodes located in the occipital and right parietal regions, referred to left mastoid $^{22,28}$. In all subjects, EEG did not show any change related to drowsiness or sleep during the experiment. Electrical stimuli of $0.2 \mathrm{~ms}$ duration were delivered to the right median nerve at the wrist, at a regular interval with a repetition rate of $3.8 \mathrm{~Hz}$. The anode was placed over the median nerve at the wrist, and the cathode $3 \mathrm{~cm}$ proximal to the anode. The stimulus intensity was set just above the motor threshold. The original broad-band SEPs were obtained using a band-pass filter of 0.5$2000 \mathrm{~Hz}$. Recording electrodes were placed on C3' $(2 \mathrm{~cm}$ posterior to C3), and Fz of the International 10-20 System. The frontal scalp region served as a ground. Electrode impedances were less than $5 \mathrm{k} \Omega$. A trial of 1000 stimuli was collected for averaging each SEP, and two successive SEPs were obtained under the same conditions to ascertain the reproducibility of SEPs ${ }^{15,20,23,27,29,30}$, and only the first recordings were used for analysis.

For separation and isolation of the HFOs from the underlying $\mathrm{N} 20$ primary cortical responses, the wide-band $(0.5-2000 \mathrm{~Hz})$ recorded responses were digitally filtered using fast reversed Fourier transformation through a band-pass of 500-1000 Hz. To determine the onset and offset of the HFO burst, we used the same method as described by Hashimoto et al: the oscillations after the onset of primary cortical response (N20) with an amplitude of twice or more than that of the background noise level were considered as the signal ${ }^{15,30}$. The noise level was measured between 9 and $15 \mathrm{~ms}$ after the stimulus ${ }^{15,30}$. The signal to noise ratio of $\mathrm{HFO}$ recordings is within the range of $20-25 \mathrm{~dB}$.

\section{Data analysis}

\section{Broad-band SEPS}

For broad-band SEPs $(0.5-2000 \mathrm{~Hz})$, we measured the latencies of N20o (N20 onset), N20p (N20 peak), P25, N33, and the peak-to-peak amplitudes of the N20o-N20p, N20p-P25, and P25-N33.

\section{HFOs}

HFOs were divided into early phase and late phase based on N20p. The HFOs from N20o to N20p were regarded as early HFOs, and the late HFOs were those later than the N20p (Figure). We then measured the maximal peak-to-baseline amplitude, the AUC (total area of positive and negative 


\section{Control subject}

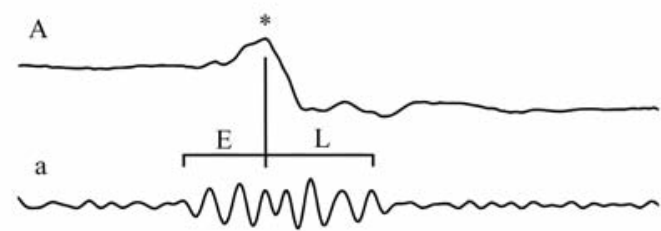

Patient: inter-ictal

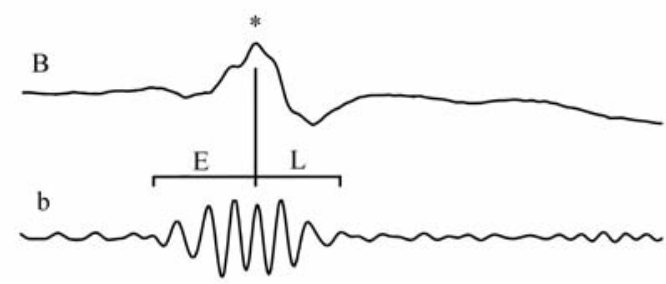

Patient: ictal
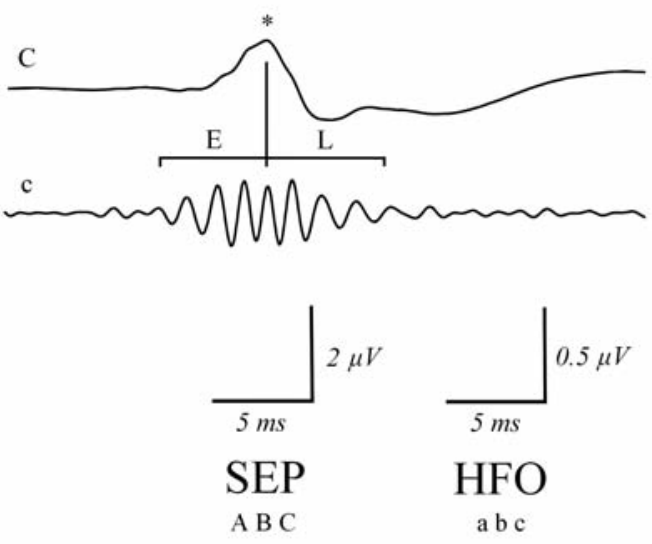

deflections from the baseline), duration, and number of wavelets of both components.

\section{Statistics}

SPSS version 13.0 for Windows (SPSS Inc., Chicago, IL, USA) was used for statistical analysis. The Student-t and chisquare tests were used for comparisons when appropriate. Oneway analysis of variance (ANOVA) followed by post-hoc least significant difference (LSD) test was used to examine the difference among the three groups: interictal, ictal, and control subjects. Statistical significance was defined as a $p$-value of $<0.05$. All tests were two-tailed.

\section{RESULTS}

We initially recruited 75 patients with migraine. Of them, 16 patients with migraine attacks one to three days prior to or post HFO recordings were excluded from analyses. The remaining 59 patients were divided into interictal $(n=35)$ and ictal $(n=24)$
Figure: Typical somatosensory evoked potentials (SEPS) and high-frequency oscillations (HFOs) pattern in control subjects and patients with migraine. High-frequency oscillations are divided into early phase and late phase based on the peak of $N 20$ (N20p) of the somatosensory evoked potential (SEP). The HFOs from onset of N2O (N2Oo) to N2Op are regarded as early HFOs, and the late HFOs are those after the N20p. There was no difference among these three individuals regarding the latencies and amplitudes of SEPs. For early HFOs, most patients with migraine whether in the interictal (b) or ictal (c) state, had significantly higher maximal amplitudes and area-undercurve (AUC) than those of the control subjects (a). However, the interictal and ictal groups did not differ in these two parameters. Besides, there was no significant difference among the three groups in duration and the number of wavelets. Regarding the late HFOs, there were no differences among the three groups across all the parameters, including the maximal amplitude, AUC, duration, and number of wavelets. Upper panel: SEP (A) and HFO (a) from a control subject. Middle panel: SEP (B) and $H F O(b)$ from a patient during interictal period. Lower panel: $S E P(C)$ and $H F O(c)$ from a patient during ictal period. *=Peak of N2O (N2Op); E: early HFOs; L: late HFOs; SEP: somatosensory evoked potential; HFO: highfrequency oscillation. groups. In the analysis sample, 39 patients had MO, and the other 20 had MA. In addition, 22 healthy, sex- and age-matched control subjects were also studied. Both patients and control subjects denied any history of systemic or other neurologic disease (except for migraine in the patient group), and their physical and neurological examinations were normal. The demographics and headache profile are shown in Table 1. In patients with migraine, the headache characteristics were similar between the ictal and interictal groups except for headache frequency (days/month) being higher in the ictal group.

\section{Broad-band SEPs}

There was no difference among the three groups (interictal, ictal, and control groups), regarding the stimulation intensity, and the latencies of N20o, N20p, P25, N33, and the peak-to-peak amplitudes of the N20o-N20p, N20p-P25, and P25-N33 (Table 2 and Figure). 
Table 1: Demographics and headache profiles of control subjects and patients with migraine

\begin{tabular}{lcccc}
\hline & Control & \multicolumn{2}{c}{ Migraine } & $p$ value \\
& & Interictal & Ictal \\
& $(\mathrm{n}=22)$ & $(\mathrm{n}=35)$ & $(\mathrm{n}=24)$ & \\
\hline Age & $31.6 \pm 6.8$ & $33.3 \pm 7.6$ & $33.8 \pm 9.7$ & 0.616 \\
Female (\%) & $15(68.2)$ & $24(68.6)$ & $16(66.7)$ & 0.988 \\
MO : MA & N/A & $22: 13$ & $17: 7$ & 0.525 \\
Disease duration (year) & N/A & $10.6 \pm 6.7$ & $13.2 \pm 9.7$ & 0.260 \\
Headache frequency (d/m) & N/A & $2.0 \pm 1.2$ & $4.2 \pm 3.5$ & 0.007 \\
Pulsating headache & N/A & $60 \%$ & $79 \%$ & 0.122 \\
Headache intensity & N/A & $60 \%$ & $67 \%$ & 0.320 \\
(\% of severe intensity) & & & & \\
\hline
\end{tabular}

MO: migraine without aura; MA: migraine with aura; N/A: not applicable; $d / m$ : days/month; \% of severe intensity: the percentage of which each migraine group (ictal and interictal) suffered from severe headache in most of their attacks

\section{HFOs}

We calculated both the maximal amplitudes and AUC of the HFOs in order to avoid the overestimation or underestimation by either means. We found the maximal amplitude and AUC were highly correlated ( $r=0.915$ for early HFO, and $r=0.859$ for late HFO, $p<0.001$, Pearson's correlation), and both calculations were good representatives of the HFO.

\section{Early HFOs}

The early HFOs results showed patients with migraine, whether in the interictal or ictal groups had significantly higher maximal amplitudes (interictal $0.190 \pm 0.091 \mu \mathrm{V}$, ictal $0.186 \pm$ $0.084 \mu \mathrm{V}$, control $0.136 \pm 0.057 \mu \mathrm{V}$, one-way ANOVA, $p=$ 0.039; post-hoc LSD: interictal vs. control, $p=0.016$; ictal vs. control, $p=0.039$ ) and AUC (interictal $293.1 \pm 124.7 \mathrm{nv} \cdot \mathrm{ms}$, ictal $304.3 \pm 147.6 \mathrm{nv} \cdot \mathrm{ms}$, control $214.6 \pm 84.2 \mathrm{nv} \cdot \mathrm{ms}$, one-way ANOVA, $p=0.029$; post-hoc LSD: interictal vs. control, $p=$ 0.022 ; ictal vs. control, $p=0.016$ ) than control subjects. However, the interictal and ictal groups did not differ in these two parameters. There was no difference among the three groups in duration and the number of wavelets of the early HFOs (Table 3, Figure).

Table 2: Latencies and amplitudes (mean \pm SD) of broad-band somatosensory evoked potential

\begin{tabular}{|c|c|c|c|c|}
\hline & \multirow{2}{*}{ Control } & \multicolumn{2}{|c|}{ Migraine } & \multirow{2}{*}{$p$ values* } \\
\hline & & Interictal & Ictal & \\
\hline Stimulation intensity (mV) & $7.7 \pm 1.6$ & $7.7 \pm 1.5$ & $8.4 \pm 2.1$ & 0.317 \\
\hline N20o latency (ms) & $16.32 \pm 0.84$ & $15.95 \pm 0.93$ & $16.01 \pm 0.90$ & 0.314 \\
\hline N20p latency (ms) & $18.94 \pm 1.03$ & $18.64 \pm 0.81$ & $18.94 \pm 1.02$ & 0.362 \\
\hline P25 latency (ms) & $24.25 \pm 2.52$ & $23.90 \pm 3.03$ & $23.67 \pm 2.29$ & 0.767 \\
\hline N33 latency (ms) & $32.43 \pm 5.24$ & $30.36 \pm 6.46$ & $30.97 \pm 5.20$ & 0.422 \\
\hline $\mathrm{N} 20 \mathrm{o}-\mathrm{N} 20 \mathrm{p}(\mu \mathrm{V})$ & $2.10 \pm 0.75$ & $2.34 \pm 1.13$ & $2.70 \pm 1.29$ & 0.179 \\
\hline N20p-P25 ( $\mu \mathrm{V})$ & $5.36 \pm 2.60$ & $5.31 \pm 3.12$ & $5.66 \pm 3.78$ & 0.911 \\
\hline P25-N33 $(\mu \mathrm{V})$ & $2.89 \pm 2.26$ & $2.68 \pm 2.32$ & $3.06 \pm 3.53$ & 0.866 \\
\hline
\end{tabular}

* One-way analysis of variance (ANOVA) test; N20o: onset of N20; N20p: peak of N20 
Table 3: All electrophysiological parameters (mean \pm SD) of HFOs

\begin{tabular}{|c|c|c|c|c|c|c|c|c|}
\hline & \multicolumn{4}{|c|}{ Early phase } & \multicolumn{4}{|c|}{ Late phase } \\
\hline & \multirow{2}{*}{ Control } & \multicolumn{2}{|c|}{ Migraine } & \multirow{2}{*}{$p$ value* } & \multirow{2}{*}{ Control } & \multicolumn{2}{|c|}{ Migraine } & \multirow{2}{*}{$p$ value* } \\
\hline & & Interictal & Ictal & & & Interictal & Ictal & \\
\hline Max peak-to-baseline amplitude $(\mu \mathrm{V})$ & $0.136 \pm 0.057^{\mathrm{a}, \mathrm{b}}$ & $0.190 \pm 0.091^{\mathrm{a}}$ & $0.186 \pm 0.084^{\mathrm{b}}$ & 0.039 & $0.151 \pm 0.069$ & $0.180 \pm 0.084$ & $0.164 \pm 0.096$ & 0.421 \\
\hline Area-under-curve (nv'ms) & $214.6 \pm 84.2^{\mathrm{a}, \mathrm{b}}$ & $293.1 \pm 124.7^{\mathrm{a}}$ & $304.3 \pm 147.6^{\mathrm{b}}$ & 0.029 & $295.7 \pm 175.9$ & $313.6 \pm 154.5$ & $321.8 \pm 229.6$ & 0.887 \\
\hline Duration of HFO (ms) & $3.26 \pm 0.48$ & $3.52 \pm 0.66$ & $3.42 \pm 0.54$ & 0.265 & $4.69 \pm 1.19$ & $4.73 \pm 1.03$ & $4.97 \pm 1.12$ & 0.638 \\
\hline Number of wavelets of HFO & $2.34 \pm 0.32$ & $2.43 \pm 0.46$ & $2.38 \pm 0.40$ & 0.717 & $3.23 \pm 0.67$ & $3.26 \pm 0.59$ & $3.40 \pm 0.66$ & 0.612 \\
\hline
\end{tabular}

* One-way analysis of variance (ANOVA) test ; ${ }_{a, b}: p<0.05$, Post-hoc least significant difference (LSD) paired comparisons; HFO: high-frequency oscillation, nv: nano-volt

\section{Late HFOs}

Regarding the late phase of the HFOs, there were no differences among the three groups in the maximal amplitude, AUC, duration, and number of wavelets (Table 3, Figure).

\section{Migraine without aura vs. migraine with aura}

Within both the ictal and interictal groups, there were no differences in all recorded parameters of both SEPs and HFOs between MA and MO patients.

\section{Discussion}

In this study, patients with migraine had higher amplitudes and AUC of early HFOs than controls. This may imply a hyperexcitability state in the subcortical regions because prior studies suggest that early HFO originates from excitatory thalamocortical fibers ${ }^{17-19,31}$. In addition, we found this hyperexcitable state may exist in both ictal and interictal periods.

It has been found during the ictal period, there is an increase in the sensitivity in the second-order (trigeminal nucleus) and third-order (thalamic relay nucleus, i.e. the ventroposterior complex, VPC) central neurons that receive convergent inputs from intracranial structures, as well as from extracranial and extracephalic structures ${ }^{32}$. Since early HFO represents the activity of deep thalamocortical fibers that originate from the VPC, an enlarged early HFO in patients with migraine during ictal period probably were derived from a sensitized hyperexcitable thalamic VPC nucleus. On the other hand, such a phenomenon has been found in both the ictal and interictal periods $^{33,34}$. Zohsel et al showed that an enhanced sensitivity to painful stimuli could be observed in patients suffering from migraine during an interictal period, even in patients with an average disease duration of 4.4 years $^{34}$. It is likely that the result of sensitization in nociceptive pain pathways can be caused by frequent pain experiences.

Studies on the subcortical regions in patients with migraine are rare. One study, by using diffusion tensor imaging, showed that patients with migraine had a lower fractional anisotropy along the thalamocortical tract ${ }^{35}$. This study may imply hyperfunctioning of the thalamocortical projections during the interictal period, in accordance with our findings.

High-frequency oscillation is a relatively new method to study the sensory pathway. However, since it explore simultaneously both the excitatory and inhibitory mechanisms of the central nervous system, it has been widely used to explore various neurological disorders, including Parkinson's disease and myoclonus epilepsy ${ }^{28}$, multiple system atrophy ${ }^{36}$, amyotrophic lateral sclerosis ${ }^{29}$, and cervical dystonia ${ }^{37}$. There are two previous studies addressing the issue on HFOs in patients with migraine. Sakuma et $\mathrm{al}^{23}$ demonstrated that the amplitudes of HFOs were significantly diminished in migraineurs at the interictal period compared to controls. Coppola et $\mathrm{al}^{24}$ found that the amplitudes and AUC of early HFOs in patients with migraine were decreased at their interictal period, but normalized at the ictal period. Regarding the late HFOs, there was no significant difference between patients and controls. They referred to these findings as a reduced preactivation excitability in migraineurs. The reasons for the discrepancies among these studies including ours are not clear. Some possible explanations are described as follows. First, the HFO settings are not completely the same among these studies (Table 4). There is an important difference between Sakuma et al's and our settings (as well as Coppola et al's), that is, the number of stimulation for average. Sakuma et al used 5000 response to generate and to average the HFOs. ${ }^{23}$ Given the stimulation rate of $4 \mathrm{~Hz}$ in their study, it would take more than 20 minutes to complete the recording. In contrast, it took four minutes in our study, and about two minutes in Coppola et al's. ${ }^{24}$ HFOs are dependent on the vigilance state ${ }^{38,39}$, and a change in the arousal state, such as eye opening or eye closing, can modify the strength of the thalamic source activity (i.e. eye opening increases and eye closing decreases thalamic activity). Prolonged recording time may cause arousal changes, which may in turn result in decreased HFO responses. However, this may not explain the discrepancy between the results from Coppola et al's and our studies. The conflicting results could be 
Table 4: Comparison of the settings and results of HFO studies on migraine patients

\begin{tabular}{|c|c|c|c|c|}
\hline & & Sakuma (23) (2004) & Coppola (24) (2005) & Lai, current study \\
\hline \multirow{6}{*}{ Stimulus Settings } & Intensity & $3 \mathrm{x}$ ST $(\sim 1 \mathrm{x} \mathrm{MT})$ & $2 \times \mathrm{MT}$ & 1x MT \\
\hline & Frequency & $3.8-4.7 \mathrm{~Hz}$ & $4.4 \mathrm{~Hz}$ & $3.8 \mathrm{~Hz}$ \\
\hline & Montage & $\mathrm{C} 3{ }^{\prime}-\mathrm{Fz}$ & $\mathrm{C} 3$ '-Fz & $\mathrm{C} 3^{\prime}-\mathrm{Fz}$ \\
\hline & Response & 5000 & 500 & 1000 \\
\hline & HFO filter & $400-800 \mathrm{~Hz}$ & $450-750 \mathrm{~Hz}$ & $500-1000 \mathrm{~Hz}$ \\
\hline & early/late discrimination & N20 peak & N20 peak & N20 peak \\
\hline \multirow{9}{*}{$\begin{array}{l}\text { Characteristics of } \\
\text { patients }\end{array}$} & Total patients, $\mathrm{N}$ & 19 & 42 & 59 \\
\hline & Female, N(\%) & $14(73.7 \%)$ & $28(66.7 \%)$ & $40(67.8 \%)$ \\
\hline & Age $($ Mean \pm SD $)$ & $38.5 \pm 17.6$ & $30.5 \pm 10.5$ & $33.5 \pm 8.4$ \\
\hline & MO:MA, N & $13: 6$ & $23: 19$ & $39: 20$ \\
\hline & $\begin{array}{l}\text { Headache frequency } \\
\text { (attacks/month) }\end{array}$ & & & \\
\hline & $0-2$ & $\mathrm{~N}=8(42 \%)$ & N/A & $\mathrm{N}=36(61 \%)$ \\
\hline & $3-4$ & $\mathrm{~N}=4(21 \%)$ & & $\mathrm{N}=16(27 \%)$ \\
\hline & $>4$ & $\mathrm{~N}=7(37 \%)$ & & $\mathrm{N}=7(12 \%)$ \\
\hline & Disease duration (years) & N/A & $\mathrm{N} / \mathrm{A}$ & $11.7 \pm 8.1$ \\
\hline \multirow{4}{*}{ Results } & Early HFO & $\downarrow$ amplitude & $\downarrow$ in both amplitude and AUC & $\uparrow$ in both amplitude and AUC \\
\hline & Late HFO & $\downarrow$ amplitude & no significance & no significance \\
\hline & Early HFO & N/A & no significance & $\uparrow$ in both amplitude and AUC \\
\hline & Late HFO & N/A & no significance & no significance \\
\hline
\end{tabular}

HFO: high-frequency oscillation, ST: sensory threshold, MT: motor threshold, MO: migraine without aura, MA: migraine with aura, N: number

due, not only completely to the different technical parameters, but also to the high variability of the subcortical and/or cortical excitability change in patients in migraine, which was also seen in the study of phosphene threshold using $\mathrm{TMS}^{40}$.

Regarding the late HFOs, there was no difference between our patient (both ictal and interictal) and control groups. Our late HFOs results are consistent with those done by Coppola et $\mathrm{al}^{24}$ although the findings of early HFOs were different. Since the late HFOs probably represent the intracortical GABAergic inhibitory activity ${ }^{20,21}$, this indicates that the inhibitory mechanism over the somatosensory cortex in patients with migraine did not differ from normal subjects.

A recent study done by Restuccia et al showed that in normal subjects, enlarged early HFOs with decreased late HFOs can be induced after slow frequency repetitive TMS (rTMS) over the primary sensory cortex ${ }^{41}$. They concluded that the inhibition of cortical excitability by slow frequency rTMS triggered an opposite, compensatory effect at the subcortical level, i.e. the increased activity of thalamocortical tract. Thus, another explanation to our finding, enlarged early HFOs in patients with migraine, might be a compensatory hyperactivity of the thalamocortical tract due to a reduced cortical excitability.
However, a major difference existed between our study and Restuccia et al's. The late HFOs (as well as SEP, discussed below) in our study showed no difference between our patient and control groups, indicating no abnormal cortical inhibition. Thus, we presumed that the enlarged early HFOs in our patient group was a primary event, instead of a compensatory phenomenon.

Regarding SEP recordings, our results were similar to those reported by these two studies, i.e. normal N20 amplitude and latency in patient and control groups ${ }^{23,24}$. Since the N20 in SEP recordings is generated by postsynaptic potentials of the pyramidal neurons in the area $3 b$ of the somatosensory cortex ${ }^{42}$, normal SEP and late HFOs in patients with migraine indicates that the somatosensory area is functionally intact.

A dissociation behavior between early HFO and SEP was observed in our findings (i.e. enlarged early HFO and normal $\mathrm{SEP}$ ). This dissociation behavior was also found in Coppola et al's study (normal N20 despite decreased or normal early HFO, in interictal and ictal period, respectively ${ }^{24}$ ). Moreover, in previous studies $^{16,21}$, the dissociation phenomenon was observed in physiologic conditions (i.e. sleep-awake cycle, interference tasks, etc.), and it was speculated accordingly that the late HFO 
was derived from discharges of intracortical GABAergic inhibitory neurons, which received thalamocortical input and exerted a feed-forward inhibition to pyramidal cells. Since the inhibitory mechanism over sensory cortex is intact as demonstrated in our and Coppola et al's studies, an enlarged ascending thalamocortical input message to pyramidal cells probably is counterbalanced by a normal-functioning inhibitory mechanism, which also received enhanced thalamocortical input. This speculation also fits well with the findings of Coppola et al's study ${ }^{24}$. In their study, normal N20 was noted despite a reduced thalamocortical input during interictal period, or a normal thalamocortical activity during ictal period. A reduced thalamocortical input may lead to a hypo-activation of both postsynaptic pyramidal cells and intracortical inhibitory neurons, resulting in a normal N20.

Another finding of our study is that in normal subjects, the early HFOs are lower in amplitude and smaller in AUC than the late HFOs. In the literature, some studies showed the same pattern as ours ${ }^{29,37}$, although some are not, i.e. larger early HFOs then the late HFOs. Interestingly, in the study conducted by Sakuma et $\mathrm{al}^{23}$, it was shown that both patterns exist in normal subjects by stimulation over right or left hands. Restuccia et al also demonstrated both patterns in their recordings ${ }^{41}$. We concluded that two patterns regarding the amplitude and AUC of early and late HFOs in normal subjects may exist, even in the same laboratory. Thus, the interpretation and comparison of HFOs across different laboratories should be careful, especially for individual data.

The study has some limitations in methodology. First, interictal and ictal recordings in the same subject were not obtained. Theoretically, a longitudinal study using paired design (interictal vs. ictal) would be more sensitive in determining the excitability changes since within-subject comparison reduces inter-subject variation. Second, one may ask if the time interval considered as the ictal period (Day $0 \pm 1$ ) in this study is optimum because excitability may vary during this period. However, if we limited our ictal group as "within 12 hours of migraine attack" as used in Coppola et al's study ${ }^{24}$, the results of early HFOs did not differ from the current results $(\mathrm{n}=12$, data not shown). Third, based on patient's recall, there was a 2.2-day difference in the monthly headache frequency between interictal and ictal groups, which may cause bias. However, the comparisons did not show any difference in the parameters of HFOs and SEP in both groups. Fourth, since migraine is a painful disorder involving cranial region, exploring the trigeminal SEP and its highfrequency components may provide more information about the pathophysiology of migraine. However, there is no wellestablished model of HFO in trigeminal SEP settings yet. Fifth, we did not simultaneously record the clinical correlate of a hyperexcitable thalamocortical pathway, i.e. the allodynic symptoms, which should be further evaluated in subsequent studies.

\section{CONCLUSIONS}

Our study showed that patients with migraine had higher amplitudes and AUC of the early-phase HFOs during interictal and ictal periods. This finding implicates subcortical hyperexcitability in patients with migraine. The N20 results did not differ between patients and controls, indicating that the ascending sensory pathway is intact. We hypothesize that the effect of enlarged early HFO might result from a sensitized ascending pathway after repeated migraine attacks. Further large-scale studies on HFOs in different headache centers may be warranted to solve the discrepancies among current studies.

\section{ACKNOWLEDGEMENT}

This study was supported in part by grants from National Science Council (95-2314-B-010-031-MY3), and Taipei Veterans General Hospital (V98C1-143), Taiwan.

\section{REFERENCES}

1. Pietrobon D, Striessnig J. Neurobiology of migraine. Nat Rev Neurosci. 2003;4(5):386-98.

2. Somjen GG. Mechanisms of spreading depression and hypoxic spreading depression-like depolarization. Physiol Rev. 2001;81 (3): 1065-96.

3. Welch KM, Barkley GL, Tepley N, et al. Central neurogenic mechanisms of migraine. Neurology. 1993;43(S3):S21-5.

4. de Tommaso M, Sciruicchio V, Tota P, et al. Somatosensory evoked potentials in migraine. Funct Neurol. 1997;12(2):77-82.

5. Firenze C, Gatto FD, Mazzotta G, et al. Somatosensory-evoked potential study in headache patients. Cephalalgia. 1988;8(3): 157-62.

6. Afra J, Cecchini A, De Pasqua V, et al. Visual evoked potentials during long periods of pattern-reversal stimulation in migraine. Brain. 1998;121(2):233-41.

7. Connolly JF, Gawel M, Rose FC. Migraine patients exhibit abnormalities in the visual evoked potential. J Neurol Neurosurg Psychiatry. 1982;45(5):464-7.

8. Aurora SK, Ahmad BK, Welch KM, et al. Transcranial magnetic stimulation confirms hyperexcitability of occipital cortex in migraine. Neurology. 1998;50(4):1111-4.

9. Khedr EM, Ahmed MA, Mohamed KA. Motor and visual cortical excitability in migraineurs patients with or without aura: transcranial magnetic stimulation. Neurophysiol Clin. 2006;36 (1):13-8.

10. Goadsby PJ. Recent advances in understanding migraine mechanisms, molecules and therapeutics. Trends Mol Med. 2007;13(1):39-44.

11. Dodick D, Silberstein S. Central sensitization theory of migraine: clinical implications. Headache. 2006;46(S4):S182-91.

12. Haas DC, Kent PF, Friedman DI. Headache caused by a single lesion of multiple sclerosis in the periaqueductal gray area. Headache. 1993;33(8):452-5.

13. Goadsby P. Neurovascular headache and a midbrain vascular malformation: evidence for a role of the brainstem in chronic migraine. Cephalalgia. 2002;22(2):107-11.

14. Cracco RQ, Cracco JB. Somatosensory evoked potential in man: far field potentials. Electroencephalogr Clin Neurophysiol. 1976;41 (5):460-6.

15. Nakano S, Hashimoto I. Comparison of somatosensory evoked high-frequency oscillations after posterior tibial and median nerve stimulation. Clin Neurophysiol. 1999;110(11):1948-52.

16. Yamada T, Kameyama S, Fuchigami Y, et al. Changes of short latency somatosensory evoked potential in sleep. Electroencephalogr Clin Neurophysiol. 1988;70(2):126-36.

17. Gobbelé R, Buchner H, Curio G. High-frequency $(600 \mathrm{~Hz})$ SEP activities originating in the subcortical and cortical human somatosensory system. Electroencephalogr Clin Neurophysiol. 1998;108(2):182-9.

18. Gobbelé R, Buchner H, Scherg M, et al. Stability of high-frequency $(600 \mathrm{~Hz})$ components in human somatosensory evoked potentials under variation of stimulus rate--evidence for a thalamic origin. Clin Neurophysiol. 1999;110(9):1659-63.

19. Gobbelé R, Waberski TD, Simon H, et al. Different origins of lowand high-frequency components $(600 \mathrm{~Hz})$ of human somatosensory evoked potentials. Clin Neurophysiol. 2004;115 (4):927-37. 
20. Hashimoto I, Mashiko T, Imada T. Somatic evoked high-frequency magnetic oscillations reflect activity of inhibitory interneurons in the human somatosensory cortex. Electroencephalogr Clin Neurophysiol. 1996;100(3):189-203.

21. Ozaki I, Hashimoto I. Neural mechanisms of the ultrafast activities. Clin EEG Neurosci. 2005;36(4):271-7.

22. Restuccia D, Della Marca G, Valeriani M, et al. Influence of cholinergic circuitries in generation of high-frequency somatosensory evoked potentials. Clin Neurophysiol. 2003;114 (8):1538-48

23. Sakuma K, Takeshima T, Ishizaki K, Nakashima K. Somatosensory evoked high-frequency oscillations in migraine patients. Clin Neurophysiol. 2004;115(8):1857-62.

24. Coppola G, Vandenheede M, Di Clemente L, et al. Somatosensory evoked high-frequency oscillations reflecting thalamo-cortical activity are decreased in migraine patients between attacks. Brain. 2005;128(1):98-103.

25. Headache classification subcommittee of the international headache society. The international classification of headache disorders: 2nd ed. Cephalalgia. 2004;24(S1):S9-160.

26. Emerson RG, Sgro JA, Pedley TA, et al. State-dependent changes in the N20 component of the median nerve somatosensory evoked potential. Neurology. 1988;38(1):64-8.

27. Urasaki E, Genmoto T, Yokota A, et al. Effects of general anesthesia on high-frequency oscillations in somato-sensory evoked potentials. J Clin Neurophysiol. 2006;23(5):426-30.

28. Mochizuki H, Ugawa Y, Machii K, et al. Somatosensory evoked high-frequency oscillation in Parkinson's disease and myoclonus epilepsy. Clin Neurophysiol. 1999;110(1):185-91.

29. Hamada M, Hanajima R, Terao $Y$, et al. Median nerve somatosensory evoked potentials and their high-frequency oscillations in amyotrophic lateral sclerosis. Clin Neurophysiol. 2007;118(4):877-86.

30. Nakano S, Hashimoto I. The later part of high-frequency oscillations in human somatosensory evoked potentials is enhanced in aged subjects. Neurosci Lett. 1999;276(2):83-6.

31. Curio G. Linking 600-Hz "spikelike" EEG/MEG wavelets ("sigmabursts") to cellular substrates: concepts and caveats. J Clin Neurophysiol. 2000;17(4):377-96.

32. Burstein R, Yarnitsky D, Goor-Aryeh I, et al. An association between migraine and cutaneous allodynia. Ann Neurol. 2000; 47(5):614-24.
33. Weissman-Fogel I, Sprecher E, Granovsky Y, et al. Repeated noxious stimulation of the skin enhances cutaneous pain perception of migraine patients in-between attacks: clinical evidence for continuous sub-threshold increase in membrane excitability of central trigeminovascular neurons. Pain. 2003; 104(3):693-700.

34. Zohsel K, Hohmeister J, Oelkers-Ax R, et al. Quantitative sensory testing in children with migraine: preliminary evidence for enhanced sensitivity to painful stimuli especially in girls. Pain. 2006;123(1-2):10-8

35. DaSilva AF, Granziera C, Tuch DS, et al. Interictal alterations of the trigeminal somatosensory pathway and periaqueductal gray matter in migraine. Neuroreport. 2007;18(4):301-5.

36. Inoue $\mathrm{K}$, Hashimoto I, Nakamura S. High-frequency oscillations in human posterior tibial somatosensory evoked potentials are enhanced in patients with Parkinson's disease and multiple system atrophy. Neurosci Lett. 2001;297(2):89-92.

37. Inoue $\mathrm{K}$, Hashimoto I, Shirai $\mathrm{T}$, et al. Disinhibition of the somatosensory cortex in cervical dystonia-decreased amplitudes of high-frequency oscillations. Clin Neurophysiol. 2004;115(7): 1624-30.

38. Gobbelé R, Waberski TD, Kuelkens S, et al. Thalamic and cortical high-frequency $(600 \mathrm{~Hz})$ somatosensory-evoked potential (SEP) components are modulated by slight arousal changes in awake subjects. Exp Brain Res. 2000;133(4):506-13.

39. Restuccia D, Della Marca G, Valeriani M, et al. Brain-stem components of high-frequency somatosensory evoked potentials are modulated by arousal changes: nasopharyngeal recordings in healthy humans. Clin Neurophysiol. 2004;115(6):1392-8.

40. Antal A, Arlt S, Nitsche M, et al. Higher variability of phosphene thresholds in migraineurs than in controls: a consecutive transcranial magnetic stimulation study. Cephalalgia. 2006;26 (7):865-70.

41. Restuccia D, Ulivelli M, De Capua A, et al. Modulation of highfrequency $(600 \mathrm{~Hz})$ somatosensory-evoked potentials after rTMS of the primary sensory cortex. Eur J Neurosci. 2007; 26(8):2349-58.

42. Allison T, McCarthy G, Wood CC, et al. Potentials evoked in human and monkey cerebral cortex by stimulation of the median nerve: a review of scalp and intracranial recordings. Brain. 1991;114(6):2465-503. 